\title{
Economical aspects of intraoperative blood loss in a large spinal surgical ward in 2018
}

\author{
Desirée Schwetje* \\ Department of Orthopedics and traumatology, University Hospital Bonn, Bonn, Germany
}

\begin{abstract}
Background: Major procedures in spine are accompanied by elevated intraoperative blood loss which often results in the need of blood transfusions. A prolonged hospital stays as well as at the intensive care unit are cost-sensible factors.

Purpose: The aim of this study was to determine a possible correlation between the intraoperative blood loss and the duration of the intensive care unit treatment, and the overall hospital stay.

Patients and Methods: This study presents a retrospective analysis of 253 patients treated in our spine surgical ward in 2018. To analyse a possible correlation the tests of Pearson's, Spearman rho's and Kendall-Tau-b's were used.

Results: The intraoperative blood loss showed a significant correlation towards the duration on the intensive care unit (ICU) in both Spearman's rho and Kendall Tau-b's. Analysis of the correlation between intraoperative blood loss and overall length of hospital stay using Pearson's showed a direct correlation with a correlation coefficient of 0.155 .
\end{abstract}

Conclusions: This study shows a correlation between intraoperative blood loss both with the duration of intensive care unit treatment and the overall hospital stay which illustrates the financial impact of elevated intraoperative blood loss.

Level of evidence: 2

\section{Introduction}

Intraoperative blood loss and blood transfusions lead to a higher mortality as well as prolonged hospital stay. Preventive measures like the use of a cell-savage or minimally invasive surgeries have been established for years in order to reduce both [1]. After various leads the tranexamic acid has proven to be efficient as a competitive synthetic lysin derivate which inhibits its binding on plasminogen and therefore fibrinolysis since $1964[2,3]$.

In orthopaedics, spine surgery is prone to long procedures with a greater blood loss [4].

Rampersaud et al. demonstrated its relevancy to health care costs because of its amount: $\$ 330$ million $(\mathrm{CDN})$ were spent in Ontario, Canada, on patient care regarding spinal conditions (including degenerative/traumatic aetiologies, outpatient visits/emergency department/imaging) [5]. Beckerman et al. showed that in singlelevel lumbar fusions $9 \%$ (lateral transpsoasfusion ("lateral"-extreme lateral $[\mathrm{XLIF}] /$ direct lateral [DLIF]/lumbar lateral [LLIF]) to $17,6 \%$ (posterolateral fusion) of overall direct health care costs were solely for room and care [6].

Therefore, cost analysis in patient care regarding the overall length of the hospital stay and frequent intensive care unit (ICU) stays of their patients is justified. This study aims to analyse a possible correlation between intraoperative blood loss and duration of the intensive care treatment (ICU) and the overall hospital stay in posterior and ventral thoracolumbar fusions, ventral cervical fusions, and thoracolumbar spinal body replacements in 2018 .

\section{Methods}

To assess the economic impact of a higher blood loss on blood transfusions, intensive care unit stay (ICU) and overall length of hospital stay in adult spine surgery we retrospectively analysed the cases treated at our level one spine centre in 2018 in adult spine surgery. Major surgical procedures such as dorsal or ventral fusion of the thoracolumbar spine, ventral cervical fusions and thoracolumbar vertebral body replacements were included because of expected blood loss. Every patient who underwent these procedures in 2018 was included and analysed retrospectively, minor spinal operations such as discectomy, dorsal selective decompression, or facet joint thermocoagulation were excluded.

To measure a possible significant relation, Pearson's, Spearman's rho and Kendall Tau-b's were used. The results are shown in linear diagrams.

Our local ethics committee's appointed number for the study is $136 / 20$ and they approved with this study.

${ }^{\star}$ Correspondence to: Desirée Schwetje, Department of Orthopedics and traumatology, University Hospital Bonn, Bonn, Germany, E-mail: Desiree. Schwetje@ukbonn.de

Key words: Spinal surgery, blood loss, intensive care unit, hospital stay, cost reduction

Received: March 17, 2021; Accepted: March 31, 2021; Published: April 07, 2021 


\section{Results}

A total of 253 patients underwent elective and urgent spinal procedures because of degeneration, scoliosis, infections (spondylodiscitis and spondylitis), cancer or fractures. In 21 patient's cancer and in 33 an acute spinal infection was the indication for surgery.

122 women and 131 men could be included with a mean age of 65,58 years.

The mean level of the procedure was 3,04 and the mean blood loss $1077.69 \mathrm{ml}$ (sum: $242480 \mathrm{ml}$; standard variation: 1.431 .41 ). Overall hospital stay was 13.52 days in average (sum: 3420 days; standard variation: 10.26) and the patients stayed a mean of 1.26 days on the intensive care unit (ICU; sum: 318 days; standard variation: 4.64). Nearly every third patient required a red blood transfusion: 83 erythrocyte transfusions (with 78 patients in total who needed a transfusion), 68 platelet transfusions.

The Pearson's correlation analysis showed no correlation between blood loss and duration of intensive care unit treatment (ICU; correlation coefficient 0.107 ).

The intraoperative blood loss showed a significant correlation towards the duration on the intensive care unit (ICU) in both Spearman's rho and Kendall Tau-b's. In Spearman's rho the correlation coefficient (between intraoperative blood loss and the stay at the intensive care unit; ICU) was calculated with 0.212 and therefore significant. The Kendall Tau-b's correlation test was calculated 0.168 , which proved to be significant as well.

To further demonstrate the calculated correlation, the two patients with treatment on the intensive care unit for more than 25 days were excluded for the next scatter diagram. Still, each patient was drawn into the correlation calculation above. The results are shown in figure 1.
Analysis of the correlation between intraoperative blood loss and overall length of hospital stay showed different results. Pearson's showed a direct correlation with a correlation coefficient of 0.155 .

To further analyse a possible correlation between intraoperative blood loss and overall length of hospital stay Spearman's and Kendall Tau-b's were used as well.

A positive correlation between intraoperative blood loss and overall hospital stay in days could be found as well. Spearmen showed a significant correlation coefficient of 0.181 and Kendall Tau-b’s 0.130 .

These results are visualized in the scatter diagram of figure 2 .

The correlation analysis between the duration of intensive care unit treatment (ICU, in days) with the overall hospital stay was significant in all three correlation tests. The Pearson's correlation coefficient was determined as 0.369 .

The Spearman's rho revealed a lower correlation coefficient but proved to be significant as well $(\mathrm{P}=0.297)$ while the Kendall tau-b's showed similar results $(\mathrm{p}=0.238)$.

Visualized in a scatter diagram in figure 3, a positive correlation between intensive care unit treatment and overall hospital stay could be confirmed.

\section{Discussion}

Elective spine surgery is prone to a large amount of blood loss resulting in frequent transfusions and a prolonged hospital stay as well as need of intensive care surveillance. Therefore, reducing the former is a possibility to decrease a patients' care expenses.

Varney and Guest examined the actual cost of blood transfusions in the United Kingdom in 2000/2001 being 898 million British pounds

\section{Scatter diagram of the intraoperative blood loss in $\mathrm{ml}$ and treatment on the intensive care unit (ICl}

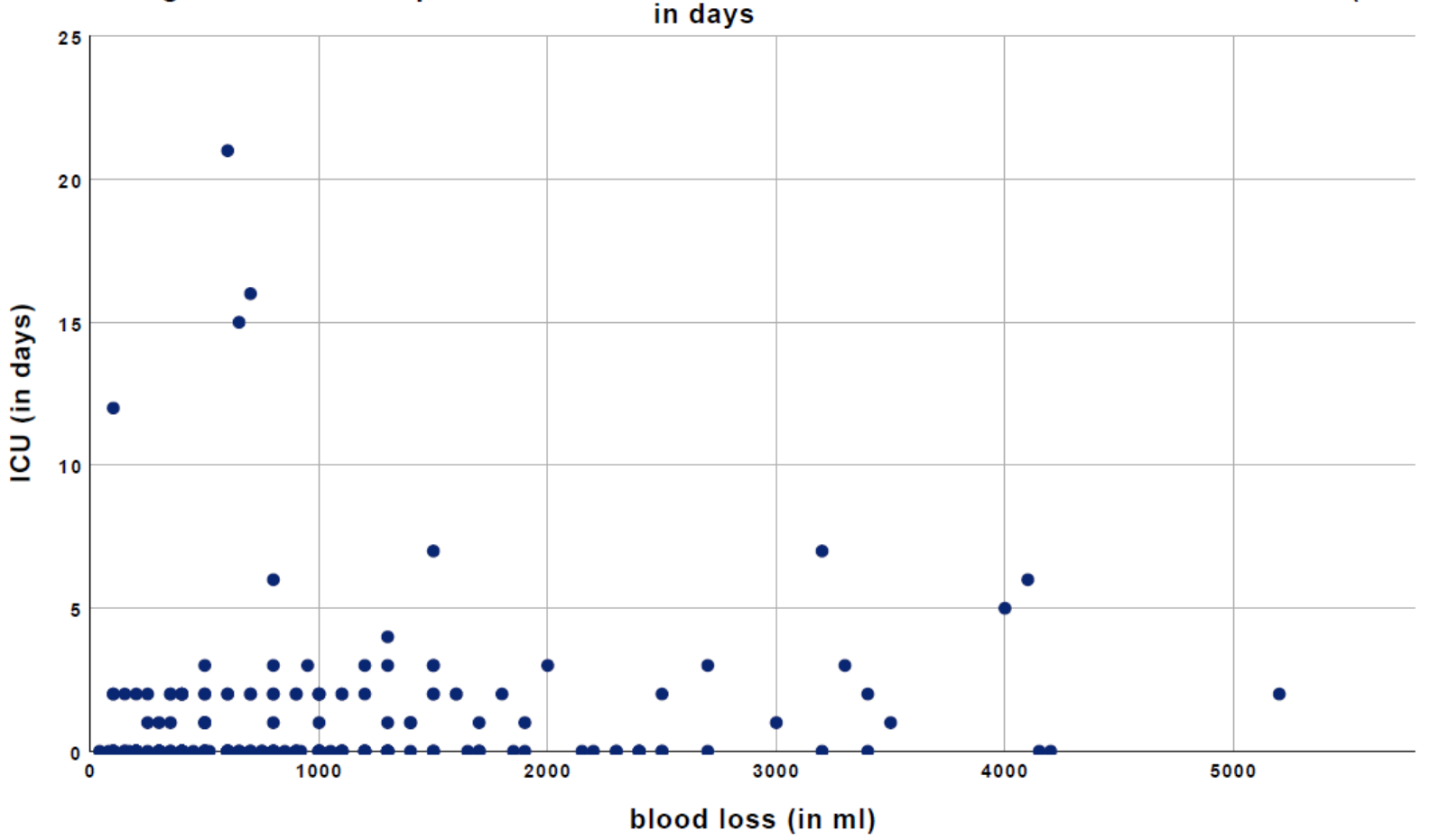

Figure 1. The scatter diagram shows the intraoperative blood loss (in $\mathrm{ml}$ ) and intensive care unit treatment (ICU, in days) excluding patients with stay on the ICU for more than 25 days 
Scatter diagram of the intraoperative blood loss in $\mathrm{ml}$ and overall hospital stay in days

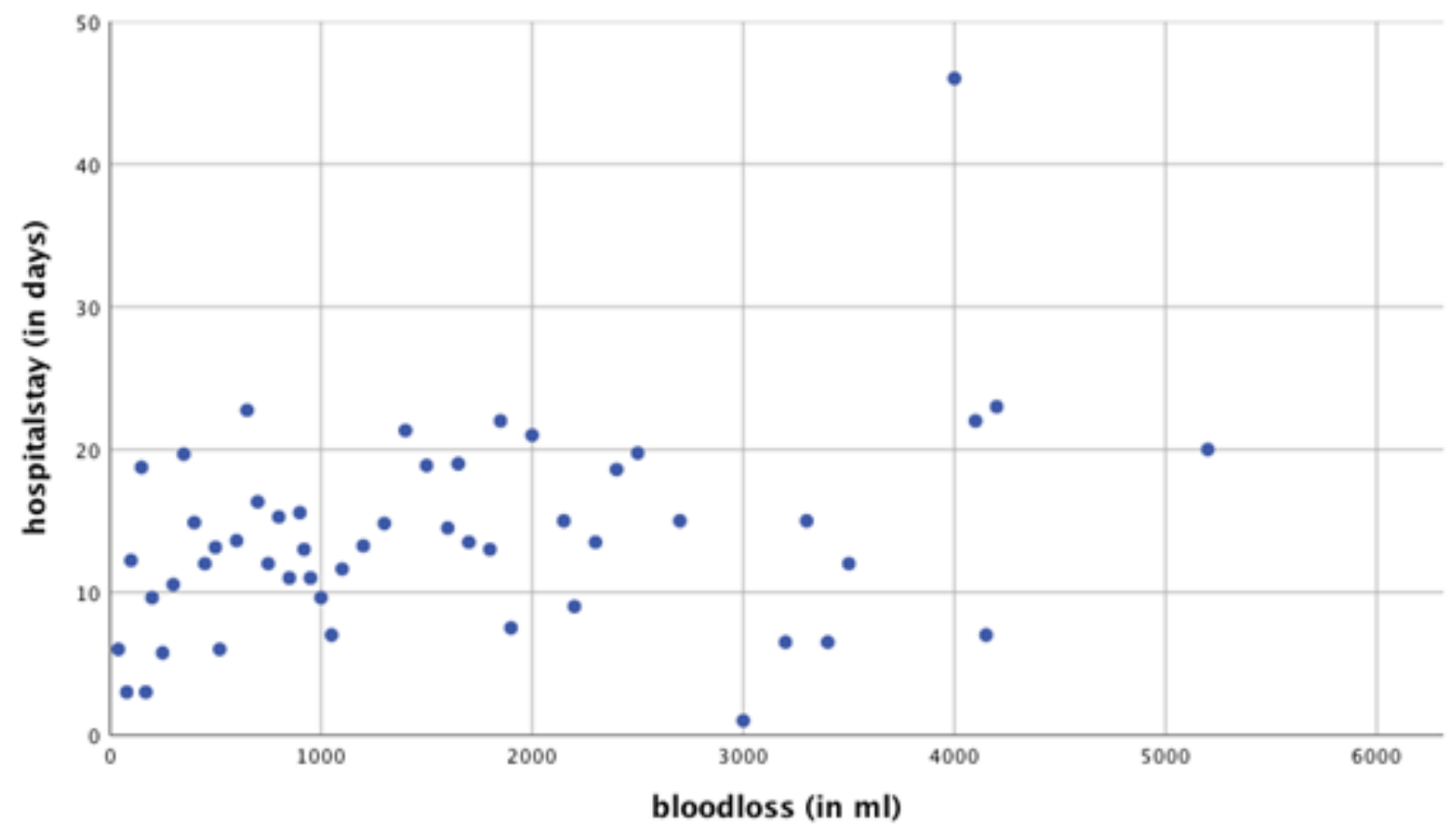

Figure 2. The scatter diagram focuses on the intraoperative blood loss (in $\mathrm{ml}$ ) and overall hospital stay (in days)

Scatter diagram of the treatment on the intensive care unit (ICU) in days and the overall hospital

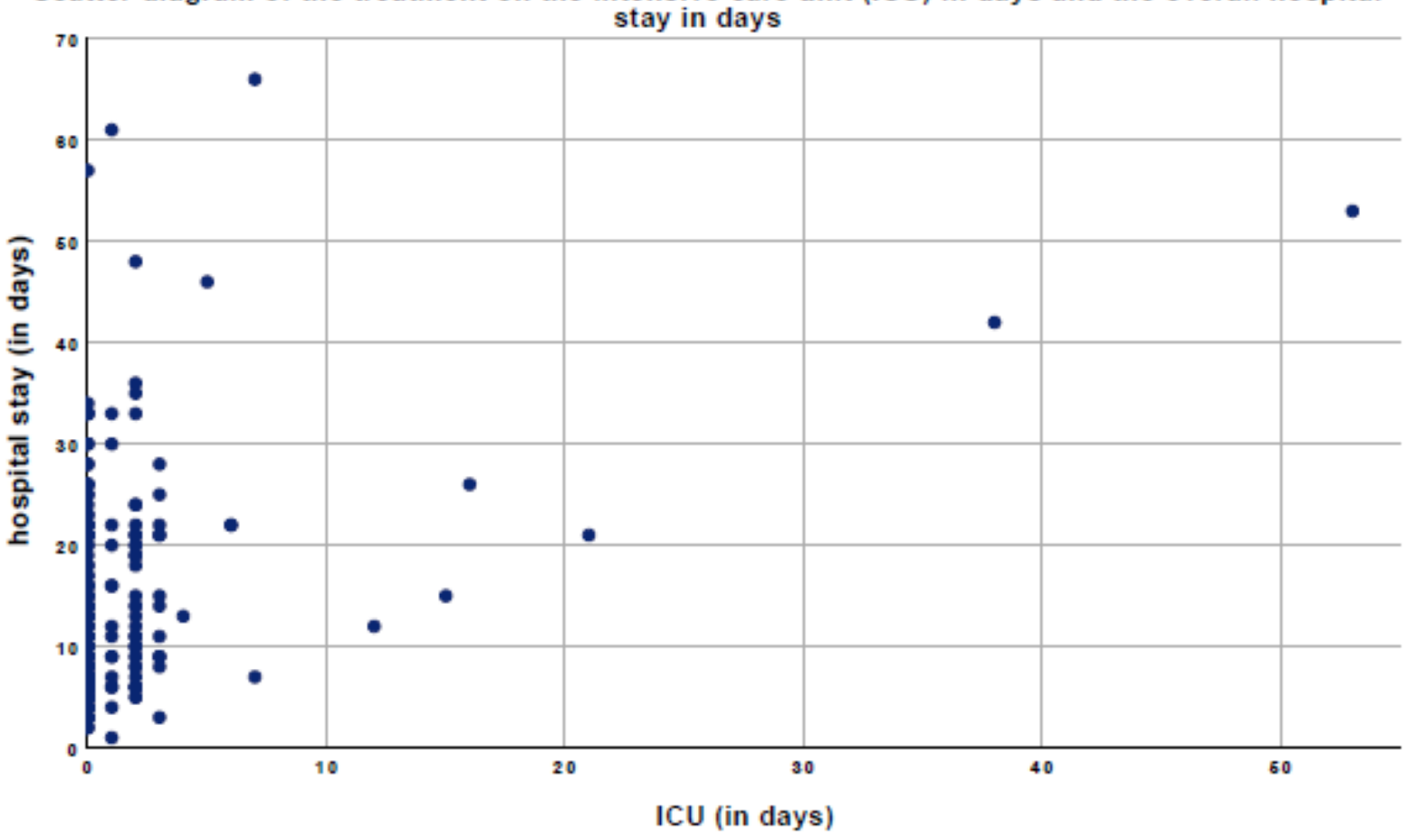

Figure 3. The scatter diagram portrays a patient's duration of intensive care unit treatment (ICU, in days) and overall hospital stay (in days) 
in total and 635 British pounds per transfusion of red blood cells, $£ 378$ for fresh frozen plasma, $£ 347$ for platelets and $£ 834$ for cryoprecipitate. When regarding the length of the hospital stay because of blood transfusions Varney and Guest found a total of 749348 adult inpatient bed days for transfusions which equal $£ 328214424$. In summary, 2000/2001 the costs of prolonged hospital stay in total were $£ 592686$ 798 [1]. In addition to direct cost for the patients 'treatment the health care system experiences an indirect loss of the donors 'productivity of

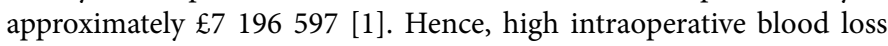
resulting in frequent blood transfusions equal a prolonged overall hospital stay as well as the indirect health care costs such as economical deficits because of the donor's absence from work for example. This stated relation could be verified by this study with a significant correlation in the Pearson's, Spearman's rho and Kendall tau-b's tests.

Dasta et al. analysed the daily costs of intensive care unit patients being the highest on day one with $\$ 6,667$ (ventilated: $\$ 10,794$ ) and the lowest from the third day ongoing at $\$ 3,184$ per day (mechanical ventilation $\$ 3,184$ ) in Ohio, United States of America. Therefore, the authors argue that reducing intensive care necessity means an overall decrease in the patients' expenses [7]. Health expanses vary among different countries and therefore data from Greece shall be mentioned as well. Karabatsou et al. described in their retrospective analysis of patients admitted to their ICU an average cost of 573.18€ per day [8]. A major criticism of this research is the lack of distinction between the admitted patients of surgical or other disciplines.

The cost per patient per day at an intensive care unit in Germany can be expected to be similar to the presented results of Greece. Regarding our patients in 2018, patients were on 1.26 days on our intensive care unit which equals nearly $722,21 €$. This calculation is made without any possibly needed blood transfusion. Our patients needed red blood cells in nearly 1 to 3 cases which equals $£ 211,67$ $(229,10 €)$ per patient. Therefore, a rounded sum of $950 €$ is a potential cost reduction if intraoperative blood loss is reduced to a minimum. This being said, other reasons for the need of postoperative observation on the ICU have to be considered. Still, a high amount of intraoperative blood loss and its impact on physical stress on heart and circulation can be assumed. As shown in our results, a significant correlation between intraoperative blood loss and duration of stay on the intensive care unit was proven by Spearman's rho and Kendall tau-b's tests (though Pearson's showed no linear correlation between these two variables). A significant correlation between treatment on the intensive care unit and overall length of the hospital stay could be observed as well but must be interpreted with caution with the first being part of the latter.

Chimento et al. argue that early mobilization is key to a good outcome after surgery. If the blood hemoglobin levels are higher the patient is more likely to take an active role in early mobilization and therefore can be discharged at an earlier stage and may experience a higher satisfaction with the result [9]. In comparison with hip or joint arthroplasty after which the patient will receive an intensive rehabilitation care the ones having been operated on their spine only receive outpatient physical therapy which is usually once or twice a week for 20 minutes in Germany. Often the waiting period for appointments result in 1-3 weeks of no therapy at all. Therefore, an early and effective inpatient physiotherapy following spine surgery is key to a better outcome and should be supported by the treating surgeon. Measures to reduce intraoperative blood loss resulting in higher blood hemoglobin levels postoperatively, such as tranexamic acid, could be a rewarding opportunity to maximize the physiotherapy's impact on the first days after the procedure. A lack of mobilization often results in a prolonged hospital stay in order to enable a discharge.
The relevancy of cost reduction can be shown by many existing publications by authors such as Lubelski et al. They developed a web tool to analyse postoperative stay and possibility of need for nonhome discharge after analyzing their patients treated for various spinal diseases in the first half of 2008 (not only the major operations as in this study). They found that age, marriage status and insurance were significantly related to an overall length and discharge disposition [10]. In combination with this paper's findings predicting and reducing a possible prolonged hospital stay can result in cost efficiency both in a direct and indirect (early mobilization) matter. Beckerman et al. found in their retrospective analysis of single-level lumbar fusion patients between 2008 and 2012 in California, United States, that 11\% of all a spinal patient's health care costs are solely room and care on a specialty orthopaedic (non-intensive care or stepdown) unit. Therefore, a reduction of the needed overall hospital stay remains a sensible opportunity to reduce expenses. Intensive care unit and stepdown ward took $1.2 \%$ of the overall treatment cost [6].

\section{Conclusion}

Major spinal procedures remain prone to high amounts of intraoperative blood loss which lead to frequent blood transfusions, intensive care treatment and prolonged overall hospital stay. This study showed a significant correlation between intraoperative blood loss and stay on the intensive care unit as well as the length of the overall hospital stay by a retrospective analysis of 253 patients treated in 2018 . Especially the correlation between an elevated intraoperative blood loss and the length of the overall hospital stay could be shown via the calculation of Pearson's, Spearman's rho and Kendall Tau-b's tests. Therefore, a reduction of intraoperative blood loss results in an overall cost reduction in health care and should be considered a sensible tool. Besides, blood loss can be viewed as an outcome parameter for the early postoperative phase.

\section{Declarations}

\section{Funding}

This research did not receive any specific grant from funding agencies in the public, commercial, or not-for-profit sectors.

\section{Conflict of interest}

The authors declare that there has been no conflict of interest or competing interest.

\section{Availability of data and material}

Data is available upon request.

\section{Ethical approval}

Our local ethics committee's appointed number for the study is $136 / 20$ and they fully approved our work.

\section{References}

1. Varney SJ, Guest JF (2003) The annual cost of blood transfusions in the UK. Transfus Med 13: 205-218. [Crossref]

2. George DA, Sarraf KM, Nwaboku H (2015) Single perioperative dose of tranexamic acid in primary hip and knee arthroplasty. Eur J Orthop Surg Traumatol 25: 129-133. [Crossref]

3. Okamoto S, Hijikata-Okunomiya A, Wanaka K, Okada Y, Okamoto U (1997) Enzymecontrolling medicines: Introduction. Semin Thromb Hemost 23: 493-501. [Crossref]

4. Li Z-J, Fu X, Xing D, Zhang H-F, Zang J-C, et al. (2013) Is tranexamic acid effective and safe in spinal surgery? A meta-analysis of randomized controlled trials. Eur Spine $J$ 22: 1950-1957. [Crossref] 
5. Rampersaud YR, Power JD, Perruccio AV, Paterson JM, Veillette C, et al. (2020) Healthcare utilization and costs for spinal conditions in Ontario, Canada - opportunities for funding high-value care: a retrospective cohort study. Spine $J$ 20: 874-881. [Crossref]

6. Beckerman D, Esparza M, Lee SI, Berven SH, Bederman SS, et al. (2020) Cost Analysis of Single-Level Lumbar Fusions. Global Spine J 10: 39-46. [Crossref]

7. Dasta JF, McLaughlin TP, Mody SH, Piech CT (2005) Daily cost of an intensive care unit day: the contribution of mechanical ventilation. Crit Care Med 33: 1266-1271. [Crossref]
8. Karabatsou D, Tsironi M, Tsigou E, Boutzouka E, Katsoulas T, et al. (2016) Variable cost of ICU care, a micro-costing analysis. Intensive Crit Care Nurs 35: 66-73. [Crossref]

9. Chimento GF, Huff T, Ochsner JL, Meyer M, Brandner L, et al. (2013) An Evaluation of the Use of Topical Tranexamic Acid in Total Knee Arthroplasty. J Arthroplasty 28: 74-77. [Crossref]

10. Lubelski D, Ehresman J, Feghali J, Tanenbaum J, Bydon A, et al. (2020) Prediction Calculator for Nonroutine Discharge and Length of Stay after Spine Surgery. Spine J 20: 1154-1158. [Crossref]

Copyright: (C2021 Schwetje D. This is an open-access article distributed under the terms of the Creative Commons Attribution License, which permits unrestricted use, distribution, and reproduction in any medium, provided the original author and source are credited. 